\title{
Zone diagrams in Euclidean spaces and in other normed spaces
}

\section{Journal Article}

\section{Author(s):}

Kawamura, Akitoshi; Matoušek, Jiří; Tokuyama, Takeshi

Publication date:

2012-12

Permanent link:

https://doi.org/10.3929/ethz-b-000058704

Rights / license:

In Copyright - Non-Commercial Use Permitted

Originally published in:

Mathematische Annalen 354(4), https://doi.org/10.1007/s00208-011-0761-1 


\title{
Zone diagrams in Euclidean spaces and in other normed spaces
}

\author{
Akitoshi Kawamura • Jiří Matoušek • \\ Takeshi Tokuyama
}

Received: 5 June 2010 / Revised: 18 December 2010 / Published online: 17 November 2011

(C) Springer-Verlag 2011

\begin{abstract}
Zone diagrams are a variation on the classical concept of Voronoi diagrams. Given $n$ sites in a metric space that compete for territory, the zone diagram is an equilibrium state in the competition. Formally it is defined as a fixed point of a certain "dominance" map. Asano, Matoušek, and Tokuyama proved the existence and uniqueness of a zone diagram for point sites in the Euclidean plane, and Reem and Reich showed existence for two arbitrary sites in an arbitrary metric space. We establish existence and uniqueness for $n$ disjoint compact sites in a Euclidean space of arbitrary (finite) dimension, and more generally, in a finite-dimensional normed space with a smooth and rotund norm. The proof is considerably simpler than that of Asano et al. We also provide an example of non-uniqueness for a norm that is rotund but not smooth. Finally, we prove existence and uniqueness for two point sites in the plane with a smooth (but not necessarily rotund) norm.
\end{abstract}

\footnotetext{
A. Kawamura

Department of Computer Science, University of Tokyo, 7-3-1 Hongo,

Bunkyo-ku, Tokyo 113-8656, Japan

J. Matoušek $(\varangle)$

Department of Applied Mathematics, Institute of Theoretical Computer Science (ITI), Charles University, Malostranské nám. 25, 11800 Praha 1, Czech Republic

e-mail: matousek@kam.mff.cuni.cz

J. Matoušek

Institute of Theoretical Computer Science, ETH Zürich, 8092 Zürich, Switzerland

T. Tokuyama

Graduate School of Information Sciences, Tohoku University, Aramaki Aza Aoba, Aoba-ku, Sendai 980-8579, Japan
} 


\section{Introduction}

Zone diagrams are a metric notion somewhat similar to the classical concept of Voronoi diagrams $[4,11]$. Let $\left(X\right.$, dist) be a metric space and let $\mathbf{P}=\left(P_{1}, \ldots, P_{n}\right)$ be an $n$-tuple of nonempty subsets of $X$ called the sites. To avoid unpleasant trivialities, we will always assume in this paper that the sites are closed and pairwise disjoint.

A zone diagram of the $n$-tuple $\mathbf{P}$ is an $n$-tuple $\mathbf{R}=\left(R_{1}, \ldots, R_{n}\right)$ of subsets of $X$, called the regions of the zone diagram, with the following defining property: Each $R_{i}$ consists of all points $x \in X$ that are closer (non-strictly) to $P_{i}$ than to the union $\bigcup_{j \neq i} R_{j}$ of all the other regions.

Informally, one can imagine that the sites compete for territory, and a site $P_{i}$ can defend only a territory $R_{i}$ that is closer to $P_{i}$ than to all the territories of the other sites. A zone diagram is an equilibrium state in this "war game".

Figure 1 shows a zone diagram in the Euclidean plane whose sites are points and segments. While in the Voronoi diagram the regions partition the whole space, in a zone diagram the union of the regions typically has a nonempty complement, called the neutral zone.

The definition of a zone diagram is implicit, since each region is determined in terms of the remaining ones. So neither existence nor uniqueness of the zone diagram is obvious, and so far only partial results in this direction have been known.

Asano et al. [2] introduced the notion of a zone diagram, for the case of $n$ point sites in the Euclidean plane, and in this setting they proved existence and uniqueness. The proof involves a case analysis specific to $\mathbb{R}^{2}$. Chun et al. [7] showed the existence and uniqueness of the two-site zone diagram in Euclidean plane when one site is a point and the other is a line. Reem and Reich [12] established, by a simple and elegant argument, the existence of a zone diagram for two sites in an arbitrary metric space (and even in a still more general setting, which they call $m$-spaces).

On the negative side, they gave an example of a three-point metric space in which the zone diagram of two point sites is not unique; thus, uniqueness needs additional

Fig. 1 A zone diagram of points and segments

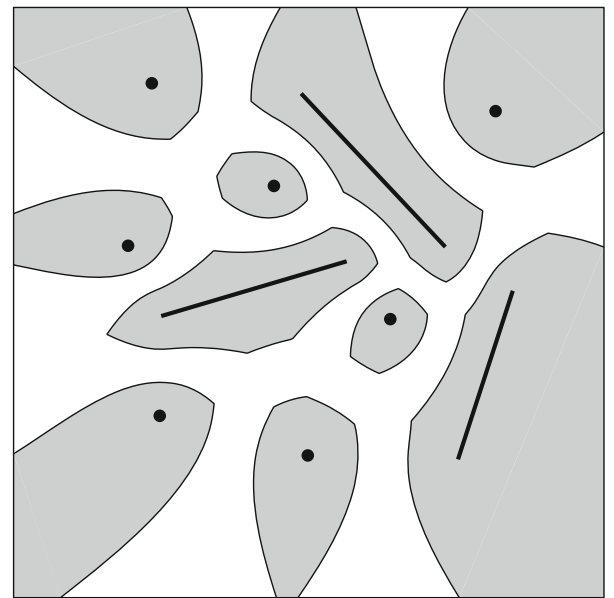


assumptions. On the other hand, for all we know, it is possible that a zone diagram always exists, for arbitrary sites in an arbitrary metric space.

\subsection{Arbitrary sites in Euclidean spaces}

In this paper, we establish the existence and uniqueness of zone diagrams in Euclidean spaces. This generalizes the main result of [2] with a considerably simpler argument. For the case of two point sites in the plane, we also obtain a new and simpler proof of the existence and uniqueness of the distance trisector curve considered by Asano et al. [3].

Theorem 1.1 Let the considered metric space $\left(X\right.$, dist) be $\mathbb{R}^{d}$ with the Euclidean distance. For every $n$-tuple $\mathbf{P}=\left(P_{1}, \ldots, P_{n}\right)$ of nonempty closed sites in $\mathbb{R}^{d}$ such that $\operatorname{dist}\left(P_{i}, P_{j}\right)>0$ for every $i \neq j$, there exists exactly one zone diagram $\mathbf{R}$.

The full proof is contained in Sects. 2 (general preliminaries) and 3. The same proof yields existence and uniqueness also for infinitely many sites in $\mathbb{R}^{d}$, provided that every two of them have distance at least $\varepsilon$ (for some fixed $\varepsilon>0$ ). Moreover, with some extra effort it may be possible to extend the proof to compact sites in a Hilbert space, for example, but in this paper we restrict ourselves to the finite-dimensional setting.

\subsection{Normed spaces}

We also investigate zone diagrams in a more general class of metric spaces, namely, finite-dimensional normed spaces. ${ }^{1}$ Normed spaces are among the most important classes of metric spaces. Moreover, as we will see, studying arbitrary norms also sheds some light on the Euclidean case. Earlier Asano and Kirkpatrick [1] investigated distance trisector curves (which are essentially equivalent to two-site zone diagrams) of two point sites under polygonal norms in the plane, obtaining results for the Euclidean case through approximation arguments.

For us, a crucial observation is that the uniqueness of zone diagrams does not hold for normed spaces. Let us consider $\mathbb{R}^{2}$ with the $\ell_{1}$ norm $\|\cdot\|_{1}$, given by $\|x\|_{1}=\left|x_{1}\right|+\left|x_{2}\right|$. It is easy to check that the two point sites $(0,0)$ and $(0,3)$ have at least two different zone diagrams, as drawn in Fig. 2. This example was essentially contained already in Asano and Kirkpatrick [1], although in a different context.

The $\ell_{1}$ norm differs from the Euclidean norm in two basic respects: the unit ball has sharp corners and straight edges; in other words, the $\ell_{1}$ norm is neither smooth nor rotund.

\footnotetext{
${ }^{1}$ A finite-dimensional (real) normed space can be thought of as the real vector space $\mathbb{R}^{d}$ with some norm, which is a mapping that assigns a nonnegative real number $\|x\|$ to each $x \in \mathbb{R}^{d}$ so that $\|x\|=0$ implies $x=0,\|\alpha x\|=|\alpha| \cdot\|x\|$ for all $\alpha \in \mathbb{R}$, and the triangle inequality holds: $\|x+y\| \leq\|x\|+\|y\|$. Each norm $\|\cdot\|$ defines a metric by $\operatorname{dist}(x, y):=\|x-y\|$. For studying a norm $\|\cdot\|$, it is usually good to look at its unit ball $\left\{x \in \mathbb{R}^{d}:\|x\| \leq 1\right\}$. The unit ball of any norm is a closed convex body $K$ that is symmetric about 0 and contains 0 in the interior. Conversely, any $K \subset \mathbb{R}^{d}$ with the listed properties is the unit ball of a (uniquely determined) norm.
} 

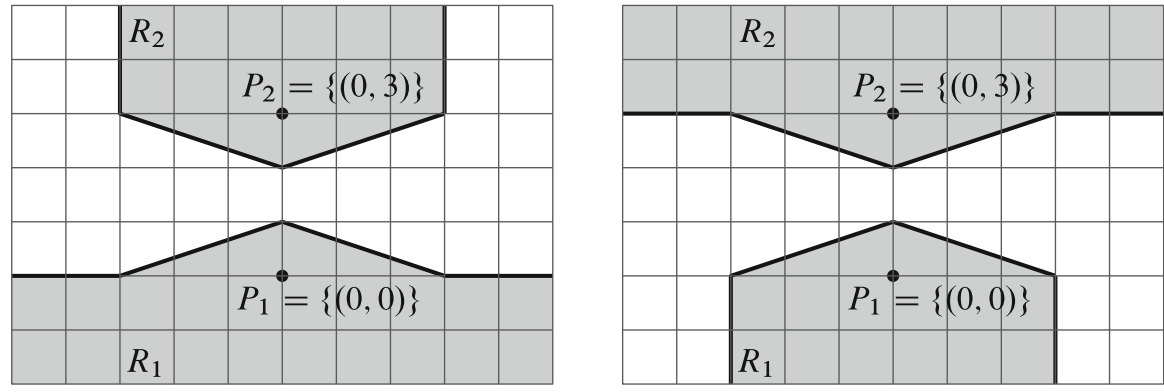

Fig. 2 Two different zone diagrams under the $\ell_{1}$ metric (drawn in the grid with unit spacing)

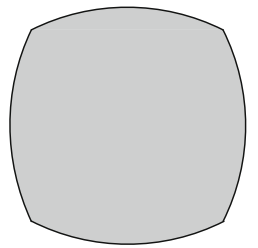

rotund but not smooth

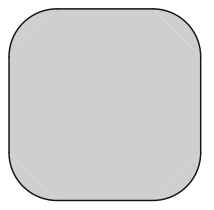

smooth but not rotund

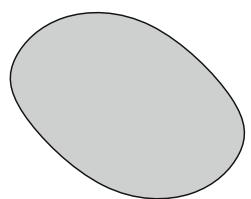

smooth and rotund

Fig. 3 Rotundity and smoothness of norms

We recall that a norm $\|\cdot\|$ on $\mathbb{R}^{d}$ is called smooth if the function $x \mapsto\|x\|$ is differentiable off the origin (geometrically, the unit ball of a smooth norm has no "sharp corners"; see Fig. 3). The property that we are actually going to use in the proof is uniform smoothness, which can be formulated as follows: The function $\rho:(0, \infty) \rightarrow[0, \infty)$ defined by

$$
\rho(t):=\sup \left\{\frac{\|u-t v\|+\|u+t v\|}{2}-1:\|u\|=\|v\|=1\right\},
$$

the modulus of smoothness, satisfies $\rho(t)=o(t)$ as $t \rightarrow 0$. A compactness argument shows that every smooth finite-dimensional Banach space is also uniformly smooth (we refer, e.g., to [5] or [8] for this and other facts on norms mentioned without proofs).

Notions dual to smoothness and uniform smoothness are rotundity and uniform convexity, respectively. A norm $\|\cdot\|$ on $\mathbb{R}^{d}$ is called rotund (or strictly convex) if for all $x, y \in \mathbb{R}^{d}$ with $\|x\|=\|y\|=1$ and $x \neq y$ we have $\left\|\frac{x+y}{2}\right\|<1$. Geometrically, the unit sphere of $\|\cdot\|$ contains no segment. A rotund norm on a finite-dimensional space is also uniformly convex, which means that for every $\varepsilon>0$ there is $\mu=\mu(\varepsilon)>0$ such that if $x, y$ are unit vectors with $\|x-y\| \geq \varepsilon$, then

$$
\left\|\frac{x+y}{2}\right\| \leq 1-\mu
$$

The Euclidean norm $\|\cdot\|_{2}$, and more generally, the $\ell_{p}$ norms with $1<p<\infty$, are both rotund and smooth. We have the following generalization of Theorem 1.1: 
Theorem 1.2 Let the considered metric space $\left(X\right.$, dist) be $\mathbb{R}^{d}$ with a norm $\|\cdot\|$ that is both smooth and rotund. For every $n$-tuple $\mathbf{P}=\left(P_{1}, \ldots, P_{n}\right)$ of nonempty closed sites in $\mathbb{R}^{d}$ such that $\operatorname{dist}\left(P_{i}, P_{j}\right)>0$ for every $i \neq j$, there exists exactly one zone diagram $\mathbf{R}$.

The proof for the Euclidean case, i.e., of Theorem 1.1, is set up so that it generalizes to smooth and rotund norms more or less immediately; there is only one lemma where we need to work harder-see Sect. 4.

Our current proof method apparently depends both on smoothness and on rotundity. In Sect. 5 we show that smoothness is indeed essential, by exhibiting a non-smooth but rotund norm in $\mathbb{R}^{d}$ with non-unique zone diagrams. On the other hand, we suspect that the assumption of rotundity in Theorem 1.2 can be dropped. Currently we have a proof (see Sect. 6) only in a rather special case:

Theorem 1.3 For two point sites $P_{0}=\left\{p_{0}\right\}$ and $P_{1}=\left\{p_{1}\right\}$ in the plane $\mathbb{R}^{2}$ with a smooth norm, there exists exactly one zone diagram.

There have been some new developments since a preliminary version of the present paper [9] has been published. Kopecká et al. [10] proved the existence of a zone diagram for every finite collection of pairwise disjoint compact sites contained in a compact convex subset of a uniformly convex normed space. (Unlike in our setting, the space can be infinite-dimensional.) The proof is based on an idea of Kopecká, briefly mentioned in Asano et al. [2], but in the general case it needs additional tricks.

de Biasi et al. [6] introduced a weakening of the notion of a zone diagram, which they call a maximal zone diagram (or a mollified zone diagram in a newer manuscript). Instead of requiring $R_{i}=\operatorname{dom}\left(P_{i}, \bigcup_{j \neq i} R_{j}\right)$ as in the definition of a zone diagram, they only want that, for each $i, R_{i} \subseteq \operatorname{dom}\left(P_{i}, \bigcup_{j \neq i} R_{j}\right)$, and moreover, the $n$-tuple $\left(R_{1}, R_{2}, \ldots, R_{n}\right)$ should be maximal w.r.t. this condition in the $\preceq$ order (see Sect. 2 below for the notation dom and $\preceq$ ). Every zone diagram is a maximal zone diagram, but not conversely, as they show by examples.

\section{Preliminaries}

Here we introduce notation and present some results from the literature, some of them in a more general context than in the original publications.

Let ( $X$, dist) be a general metric space. The closure of a set $A \subseteq X$ is denoted by $\bar{A}$, while $\partial A$ stands for its boundary. The (closed) ball of radius $r$ centered at $x$ is denoted by $\mathrm{B}(x, r)$.

For sets $A, B \subseteq X$, not both empty, we define the dominance region of $A$ over $B$ as the set

$$
\operatorname{dom}(A, B):=\{x \in X: \operatorname{dist}(x, A) \leq \operatorname{dist}(x, B)\},
$$

where

$$
\operatorname{dist}(C, D):=\inf _{x \in C, y \in D} \operatorname{dist}(x, y) \in[0,+\infty]
$$


denotes the distance between the sets $C$ and $D$.

Let us fix an $n$-tuple $\mathbf{P}=\left(P_{1}, \ldots, P_{n}\right)$ of sites, i.e., nonempty subsets of $X$ (which, as above, we assume to be disjoint and closed). For an $n$-tuple $\mathbf{R}=\left(R_{1}, \ldots, R_{n}\right)$ of arbitrary subsets of $X$, we define another $n$-tuple of regions $\mathbf{R}^{\prime}=\left(R_{1}^{\prime}, \ldots, R_{n}^{\prime}\right)$ denoted by $\mathbf{D o m} \mathbf{R}$ and given by

$$
R_{i}^{\prime}:=\operatorname{dom}\left(P_{i}, \bigcup_{j \neq i} R_{j}\right), \quad i=1, \ldots, n
$$

(the sites are considered fixed and they are a part of the definition of the operator Dom).

The definition of a zone diagram can now be expressed as follows: An $n$-tuple $\mathbf{R}$ is called a zone diagram for the $n$-tuple $\mathbf{P}$ of sites if $\mathbf{R}=\mathbf{D o m} \mathbf{R}$ (componentwise equality, i.e., $R_{i}=\operatorname{dom}\left(P_{i}, \bigcup_{j \neq i} R_{j}\right)$ for all $\left.i\right)$.

For two $n$-tuples $\mathbf{R}$ and $\mathbf{S}$ of sets, we write $\mathbf{R} \preceq \mathbf{S}$ if $R_{i} \subseteq S_{i}$ for every $i$. It is easily seen (see, e.g., [2]) that the operator Dom is antimonotone, i.e., $\mathbf{R} \preceq \mathbf{S}$ implies $\operatorname{Dom} \mathbf{R} \succeq \operatorname{Dom} \mathbf{S}$. Our starting point in the proofs of Theorems 1.1 and 1.2 is the following general result.

Theorem 2.1 For every n-tuple $\mathbf{P}$ of sites (in any metric space), there exist $n$-tuples $\mathbf{R}$ and $\mathbf{S}$ such that $\mathbf{R}=\operatorname{Dom} \mathbf{S}$ and $\mathbf{S}=\operatorname{Dom} \mathbf{R}$, and moreover, for every $n$-tuples $\mathbf{R}^{\prime}, \mathbf{S}^{\prime}$ with $\mathbf{R}^{\prime}=\operatorname{Dom} \mathbf{S}^{\prime}$ and $\mathbf{S}^{\prime}=\operatorname{Dom} \mathbf{R}^{\prime}$, we have $\mathbf{R} \preceq \mathbf{R}^{\prime}$ and $\mathbf{S}^{\prime} \preceq \mathbf{S}$ (and in particular, $\mathbf{R} \preceq \mathbf{S}$ ).

A special case of this result, for point sites in the Euclidean plane, was first proved by Asano et al. [2, Lemma 5.1], while the general case is due to Reem and Reich [12]. We recall their proof for the reader's convenience.

Proof We use the following theorem of Knaster and Tarski (see [13]): If $\mathcal{L}=(L, \preceq)$ is a complete lattice and $g: \mathcal{L} \rightarrow \mathcal{L}$ is a monotone mapping, then $g$ has at least one fixed point (i.e., $x \in L$ with $g(x)=x$ ), and moreover, there exists a smallest fixed point $x_{0}$ and a largest fixed point $x_{1}$, i.e., such that $x_{0} \preceq x \preceq x_{1}$ for every fixed point $x$.

To prove Theorem 2.1, we let $L$ be the system of all ordered $n$-tuples $\mathbf{D}$ such that $P_{i} \subseteq D_{i}$ for every $i$. It is easy to check that $\mathcal{L}=(L, \preceq)$ (where $\preceq$ is defined as above) is a complete lattice. Let $g:=\mathbf{D o m}^{2}$; that is, $g(\mathbf{D}):=\operatorname{Dom}(\operatorname{Dom} \mathbf{D})$. Then we let $\mathbf{R}$ be the smallest fixed point of $g$ as in the Knaster-Tarski theorem, and $\mathbf{S}:=\operatorname{Dom} \mathbf{R}$. Clearly $\operatorname{Dom} \mathbf{S}=\mathbf{D o m}^{2} \mathbf{R}=g(\mathbf{R})=\mathbf{R}$. Moreover, if $\mathbf{R}^{\prime}$ and $\mathbf{S}^{\prime}$ satisfy $\mathbf{R}^{\prime}=\operatorname{Dom} \mathbf{S}^{\prime}$ and $\mathbf{S}^{\prime}=\operatorname{Dom} \mathbf{R}^{\prime}$, then $\mathbf{R}^{\prime}$ and $\mathbf{S}^{\prime}$ are both fixed points of Dom ${ }^{2}$, and thus $\mathbf{R} \preceq \mathbf{R}^{\prime}, \mathbf{S}^{\prime} \preceq \mathbf{S}$ as claimed.

Let us remark that the earlier proof by Asano et al. [2] can easily be extended so that it yields the special case of Theorem 2.1 where the metric space is a finite-dimensional normed space with a rotund norm (and the sites are arbitrary). This is discussed in more detail in an ArXiv version of the present paper [9]. That proof is still of some interest, since it is more "constructive" than the one shown above, in that the alleged $n$-tuples $\mathbf{R}$ and $\mathbf{S}$ are obtained by an iterative process. 
We finish this section with a simple geometric lemma. It was used, in a less general setting, in [2] (proof of Lemma 4.3).

Observation 2.2 Let $\mathbf{P}$ be an $n$-tuple of sites (in an arbitrary metric space), and suppose that $\varepsilon:=\min _{i \neq j} \operatorname{dist}\left(P_{i}, P_{j}\right)>0$ and that $\mathbf{R}$ and $\mathbf{S}$ satisfy $\mathbf{R}=\operatorname{Dom} \mathbf{S}$ and $\mathbf{S}=\operatorname{Dom} \mathbf{R}$. Then $\operatorname{dist}\left(P_{i}, \bigcup_{j \neq i} S_{j}\right) \geq \frac{\varepsilon}{2}$, and consequently, the $\frac{\varepsilon}{4}$-neighborhood of each $P_{i}$ is contained in $R_{i}$.

Proof We recall the simple proof from [2]. We first note that $\mathbf{V}=\left(V_{1}, \ldots, V_{n}\right):=$ Dom $\mathbf{P}$ is the classical Voronoi diagram of $\mathbf{P}$, and the open $\frac{\varepsilon}{2}$-neighborhood of $P_{i}$ does not intersect $\bigcup_{j \neq i} V_{j}$. Since $\mathbf{P} \preceq \mathbf{R}$, we have $\operatorname{Dom} \mathbf{P} \succeq \operatorname{Dom} \mathbf{R}=\mathbf{S}$, and hence the open $\frac{\varepsilon}{2}$-neighborhood of $P_{i}$ is disjoint from $\bigcup_{j \neq i} S_{j}$ as well, as claimed.

\section{The Euclidean case}

Here we prove Theorem 1.1; throughout this section, dist denotes the Euclidean distance. In addition to Theorem 2.1 and Observation 2.2, we also need the next lemma.

Lemma 3.1 (Cone lemma, Euclidean case) Let $\mathbf{P}$ be an $n$-tuple of (nonempty closed) sites in $\mathbb{R}^{d}$ with the Euclidean metric such that $\varepsilon:=\min _{i \neq j} \operatorname{dist}\left(P_{i}, P_{j}\right)>0$, and let $\mathbf{R}$ and $\mathbf{S}$ satisfy $\mathbf{R}=\operatorname{Dom} \mathbf{S}$ and $\mathbf{S}=\operatorname{Dom} \mathbf{R}$. Let a be a point of some $R_{i}$, and let $p \in P_{i}$ be a point of the corresponding site closest to a (such a nearest point exists by compactness). Then the set

$$
K:=\operatorname{conv}\left(\{a\} \cup \mathrm{B}\left(p, \frac{\varepsilon}{4}\right)\right)
$$

is contained in $R_{i}$; see Fig. 4.

The following proof is rather specific for the Euclidean metric (the lemma fails for the $\ell_{1}$ metric, for example).

Proof Both $a$ and $\mathrm{B}\left(p, \frac{\varepsilon}{4}\right)$ are contained in $\operatorname{dom}\left(p, \bigcup_{j \neq i} S_{j}\right)$ (the latter by Observation 2.2). For the Euclidean metric, the dominance region of a point over any set is convex, since it is the intersection of halfspaces. Hence $K \subseteq \operatorname{dom}\left(p, \bigcup_{j \neq i} S_{j}\right) \subseteq R_{i}$.

Now we describe the general strategy of the proof of Theorem 1.1. With $\mathbf{R}$ and $\mathbf{S}$ as in Theorem 2.1, it suffices to prove $\mathbf{R}=\mathbf{S}$. For contradiction, we assume that it not the case, i.e., that $R:=\bigcup_{i=1}^{n} R_{i}$ is properly contained in $S:=\bigcup_{i=1}^{n} S_{i}$; see the schematic illustration in Fig. 5.

Fig. 4 The cone $K$

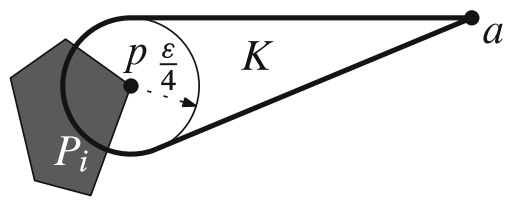




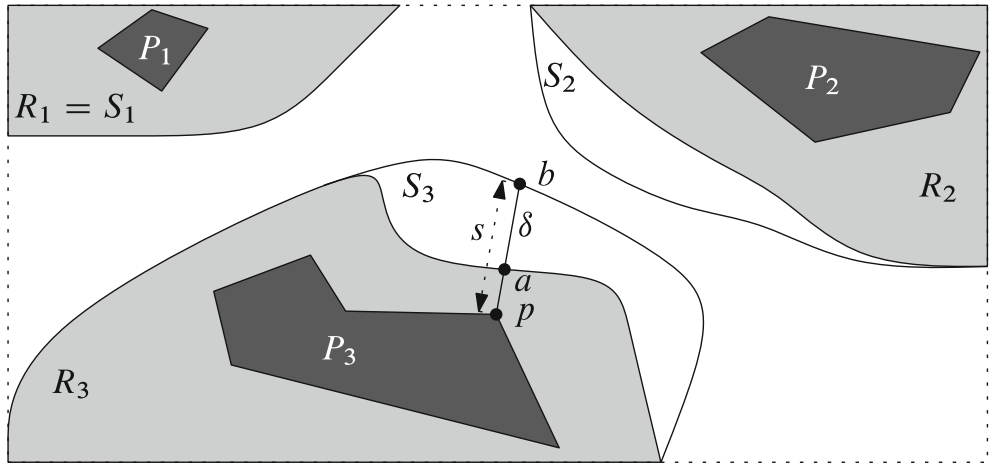

Fig. 5 The setting of the proof of Theorem 1.1 (a schematic picture)

For a point $b \in S \backslash \bigcup_{i=1}^{n} P_{i}$, let $s(b):=\operatorname{dist}\left(b, P_{i}\right)$ be the distance from the nearest site $P_{i}$, and let $p=p(b) \in P_{i}$ be a point where this distance is attained. Let $a=a(b)$ be the closest point to $b$ that lies in the intersection of $R_{i}$ with the segment $b p$. It is easily seen, using the triangle inequality, that $p$ is also a nearest point of $P_{i}$ to $a$. Thus, the set $K$ in Lemma 3.1 is contained in $R_{i}$, and in particular, $a$ is the only intersection of the segment $b p$ with $\partial R_{i}$. We set $\delta(b):=\operatorname{dist}(b, a)$. The parameters $s(b)$ and $\delta(b)$ will measure, in some sense, how much $S$ differs from $R$ "at $b$ ".

Assuming $\mathbf{R} \neq \mathbf{S}$, we choose a point $b_{0} \in S \backslash R$. Then, using $b_{0}$, we find $b_{1} \in S \backslash R$ where $S$ differs from $R$ "more than" at $b_{0}$. Iterating the same procedure we obtain an infinite sequence $b_{0}, b_{1}, b_{2}, b_{3}, \ldots$ of points, and the difference will "grow" beyond bounds, while, on the other hand, it has to stay bounded - and this way we reach a contradiction.

More concretely, for every integer $t \geq 1$ we will construct $b_{t}$ from $b_{t-1}$ so that, with $s:=s\left(b_{t-1}\right), s^{\prime}:=s\left(b_{t}\right), \delta:=\delta\left(b_{t-1}\right)$, and $\delta^{\prime}:=\delta\left(b_{t}\right)$, we have

(A) $s^{\prime} \leq s-\alpha$, or

(B) $s^{\prime} \leq s-\delta$ and $\delta^{\prime} \geq \delta$,

where $\alpha>0$ is a constant that depends on $s_{0}:=s\left(b_{0}\right)$ and $\varepsilon$, but not on $t$.

Thus, as $t$ increases, $s\left(b_{t}\right)$ keeps decreasing. Since $s\left(b_{t}\right)$ is bounded from below by $\frac{\varepsilon}{4}$ by Observation 2.2 , case (A) can happen only finitely many times. Therefore, from some $t$ on, we have case (B) only. But this also causes $s\left(b_{t}\right)$ to decrease towards 0 - $\mathrm{a}$ contradiction.

It remains to describe the construction of $b_{t}$ from $b_{t-1}$, and this is done in the next lemma.

Lemma 3.2 For every $s_{0}$ and $\varepsilon>0$ there exists $\alpha>0$ such that if $b \in S \backslash R$ satisfies $s:=s(b) \leq s_{0}$, then there exists another point $b^{\prime} \in S \backslash R$ such that $s^{\prime}:=s\left(b^{\prime}\right), \delta:=$ $\delta(b)$ and $\delta^{\prime}:=\delta\left(b^{\prime}\right)$ satisfy $(\mathrm{A})$ or $(\mathrm{B})$.

Proof Let $b \in S_{i}$, let $a:=a(b), p:=p(b)$, and write $r=\operatorname{dist}(a, p)$; see Fig. 6 . Since $a \in \partial R_{i}$ and $\mathbf{R}=\operatorname{Dom} \mathbf{S}$, there exist $j \neq i$ and $b^{\prime} \in S_{j}$ with $\operatorname{dist}\left(a, b^{\prime}\right)=r$. If there are several possible $b^{\prime}$, we choose one of them arbitrarily. 


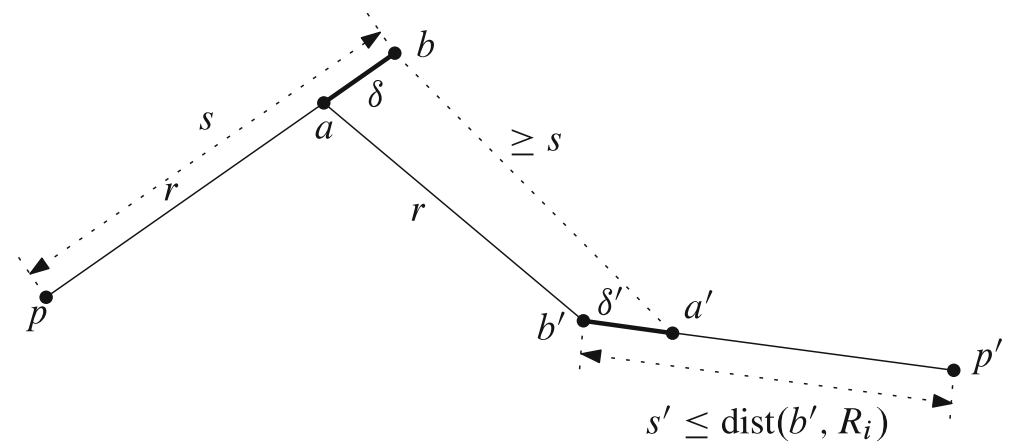

Fig. 6 The construction of $b^{\prime}$

First we check that $b^{\prime} \notin R$, or in other words, that $\delta^{\prime}>0$. During this step we also derive a lower bound for $\delta^{\prime}$ that will be useful later. Since $b \in S, a^{\prime} \in R$, and $\mathbf{S}=\operatorname{Dom} \mathbf{R}$, we have $\operatorname{dist}\left(a^{\prime}, b\right) \geq s$. Then we bound, using the triangle inequality,

$$
\delta^{\prime} \geq \operatorname{dist}\left(a^{\prime}, b\right)-\operatorname{dist}\left(b, b^{\prime}\right) \geq s-\operatorname{dist}\left(b, b^{\prime}\right) .
$$

Supposing for contradiction that $\delta^{\prime}=0$, we get $\operatorname{dist}\left(b, b^{\prime}\right) \geq s$. But the triangle inequality gives $\operatorname{dist}\left(b, b^{\prime}\right) \leq \operatorname{dist}(b, a)+\operatorname{dist}\left(a, b^{\prime}\right)=r+\delta=s$, and hence the triangle inequality here holds with equality. For the Euclidean metric, this can happen only if $a$ lies on the segment $b b^{\prime}$, and then $b^{\prime}$ has to coincide with $p$, which is impossible. So $\delta^{\prime}>0$ indeed.

Next, since $\mathbf{S}=\operatorname{Dom} \mathbf{R}$ and $b^{\prime} \in S$, we have $s^{\prime} \leq \operatorname{dist}\left(b^{\prime}, R_{i}\right)$. An obvious upper bound for $\operatorname{dist}\left(b^{\prime}, R_{i}\right)$ is $\operatorname{dist}\left(b^{\prime}, a\right)=r=s-\delta$, and thus the first inequality in (B), namely, $s^{\prime} \leq s-\delta$, always holds.

Moreover, if $\delta \geq \alpha$, then $s^{\prime} \leq s-\delta \leq s-\alpha$, and we have (A). For the rest of the proof we thus assume that $\delta<\alpha$ (where $\alpha$ hasn't been fixed yet-so far we're free to choose it as a positive function of $\varepsilon$ and $s_{0}$ in any way we like).

Let us consider the ball $\mathrm{B}\left(b^{\prime}, r\right)$; see Fig. 7. If it contains $b$, as in the left picture, we have $\operatorname{dist}\left(b^{\prime}, b\right) \leq r$, and thus by (2) we have $\delta^{\prime} \geq s-r=\delta$. Then (B) holds. Thus, the last case to deal with is $b \notin \mathrm{B}\left(b^{\prime}, r\right)$.

Let us consider the cone $K=\operatorname{conv}\left(\{a\} \cup \mathrm{B}\left(p, \frac{\varepsilon}{4}\right)\right)$ as in Lemma 3.1. Its opening angle $\gamma$ is bounded away from 0 in terms of $\varepsilon$ and $s_{0}$; indeed, we have $\gamma \geq \varepsilon / 4 s_{0}$ (here and in the further steps we use that $r$ and $s$ are bounded from below by $\varepsilon$ and from above by $s_{0}$ ).

Let $\Pi$ be a 2 -dimensional plane containing $p, a, b^{\prime}$; it also contains $b$ since $p, a, b$ are collinear. Let $k$ be the ray originating at $a$ and containing $b$, and let $l$ be the ray in $\Pi$ originating at $a$ and making the angle $\pi-\frac{\gamma}{2}$ with $k$ (on the side of $b^{\prime}$ ); see Fig. 7 right.

Since the angle between the rays $k$ and $l$ is bounded away from the straight angle, the Euclidean ball $\mathrm{B}\left(b^{\prime}, r\right)$ cuts a segment of significant length from at least one of these rays. This length can be bounded from below by a positive quantity $\beta$ depending only on $s_{0}$ and $\varepsilon$. Indeed, in the extreme case where $k$ and $l$ make the same 

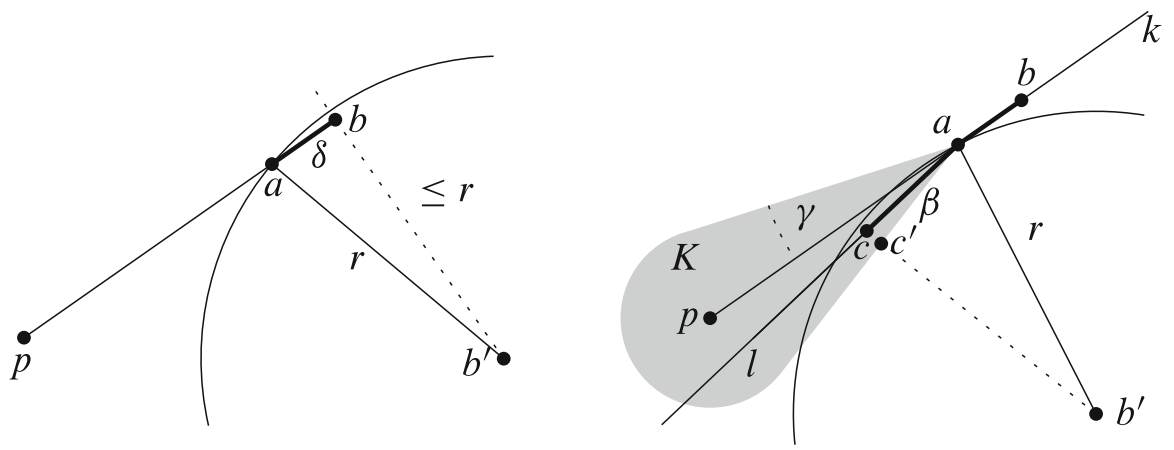

Fig. 7 The $r$-ball around $b^{\prime}$

Fig. 8 Bounding the quantity $\beta$ from below in the Euclidean case

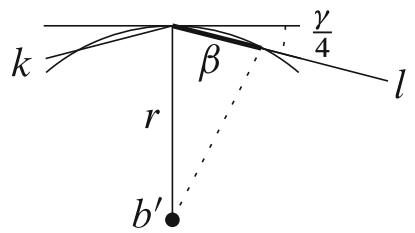

angle $\frac{\gamma}{4}$ with the tangent of $\mathrm{B}\left(b^{\prime}, r\right)$, shown in Fig. 8, easy trigonometry yields $\beta=$ $2 r \sin \frac{\gamma}{4}$.

So far we have not fixed $\alpha$, and so now we can make sure that $\alpha<\beta$. Since we assume $b \notin \mathrm{B}\left(b^{\prime}, r\right)$, the segment of length $\beta$ cut out by $\mathrm{B}\left(b^{\prime}, r\right)$ can't belong to the ray $k$. So the situation is as in Fig. 7 right: $\mathrm{B}\left(b^{\prime}, r\right)$ contains the initial segment $a c$ of $l$ of length $\beta$. Hence $\operatorname{dist}\left(b^{\prime}, c\right) \leq r$.

The distance $\operatorname{dist}\left(c, \mathbb{R}^{d} \backslash K\right)$ is bounded away from 0 in terms of $\beta$ and $\gamma$, and so we may fix $\alpha$ so that $\operatorname{dist}\left(c, \mathbb{R}^{d} \backslash K\right) \geq \alpha$.

Let $c^{\prime}$ be the point where the segment $b^{\prime} c$ meets the boundary of $K$. We have

$\operatorname{dist}\left(b^{\prime}, K\right) \leq \operatorname{dist}\left(b^{\prime}, c^{\prime}\right)=\operatorname{dist}\left(b^{\prime}, c\right)-\operatorname{dist}\left(c, c^{\prime}\right) \leq r-\operatorname{dist}\left(c, \mathbb{R}^{d} \backslash K\right) \leq r-\alpha$.

Then, finally, using $K \subseteq R_{i}$, we have

$$
s^{\prime} \leq \operatorname{dist}\left(b^{\prime}, R_{i}\right) \leq \operatorname{dist}\left(b^{\prime}, K\right) \leq r-\alpha<s-\alpha,
$$

and so (A) holds. This concludes the proof of Lemma 3.2, as well as that of Theorem 1.1.

\section{The case of smooth and rotund norms}

In this section we establish Theorem 1.2. We begin with the part where the proof differs from the Euclidean case: the cone lemma. In the Euclidean case, we used the fact that for points $p \neq q$, $\operatorname{dom}(p, q)$ is a halfspace, and consequently, $\operatorname{dom}(p, X)$ is 
Fig. 9 The dominance region of the point $(0,0)$ against $(2,1)$ in the $\ell_{4}$ norm

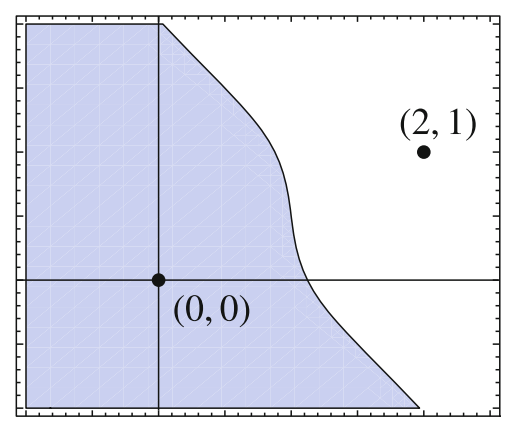

Fig. 10 The dominance region of a point against a halfspace
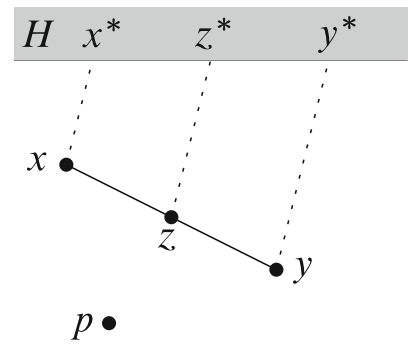

convex for arbitrary $X$. For other norms $\operatorname{dom}(p, q)$ need not be convex, though; see Fig. 9.

We have at least the following convexity result.

Lemma 4.1 Let us consider $\mathbb{R}^{d}$ with an arbitrary norm $\|\cdot\|$, let $H$ be a closed halfspace, and let $p \notin H$ be a point. Then $\operatorname{dom}(p, H)$ is convex.

Consequently, if the complement of a closed set $A \subseteq \mathbb{R}^{d}$ is convex and $p \notin A$, then $\operatorname{dom}(p, A)$ is convex.

Proof Let $x \notin H$ be a point and let $x^{*} \in \partial H$ be a point where $\operatorname{dist}(x, H)$, the distance of $x$ to $H$ measured by $\|\cdot\|$, is attained. If $y \notin H$ is another point and $y^{*} \in \partial H$ is the point such that the vectors $x-x^{*}$ and $y-y^{*}$ are parallel, then $\left\|y-y^{*}\right\|=\operatorname{dist}(y, H)$; see Fig. 10.

Now let $x, y \in \operatorname{dom}(p, H)$, let $x^{*}, y^{*}$ be as above, set $z:=(x+y) / 2$, and let $z^{*}$ be defined analogously to $y^{*}$. Then we get $\operatorname{dist}(z, H)=\left\|z-z^{*}\right\|=\left(\left\|x-x^{*}\right\|+\right.$ $\left.\left\|y-y^{*}\right\|\right) / 2=(\operatorname{dist}(x, H)+\operatorname{dist}(y, H)) / 2$. From this $z \in \operatorname{dom}(p, H)$ is immediate, since $\|p-z\| \leq(\|p-x\|+\|p-y\|) / 2 \leq(\operatorname{dist}(x, H)+\operatorname{dist}(y, H)) / 2=\operatorname{dist}(z, H)$. This proves the first part of the lemma.

The second part follows easily: $A$ can be expressed as a union of closed halfspaces $H$, and $\operatorname{dom}(p, A)$ is the intersection of the convex sets $\operatorname{dom}(p, H)$.

Now we prove a cone lemma, similar to Lemma 3.1:

Lemma 4.2 (Cone lemma for rotund norms) Let $\|\cdot\|$ be a rotund norm on $\mathbb{R}^{d}$. Suppose that an n-tuple $\mathbf{P}$ of sites satisfies $\varepsilon:=\min _{i \neq j} \operatorname{dist}\left(P_{i}, P_{j}\right)>0$, and $\mathbf{R}$ and $\mathbf{S}$ satisfy $\mathbf{R}=\operatorname{Dom} \mathbf{S}$ and $\mathbf{S}=\operatorname{Dom} \mathbf{R}$. Then for every $s_{0}>0$ there is $\rho>0$ 
Fig. 11 The sets $C$ (shaded) and $D$

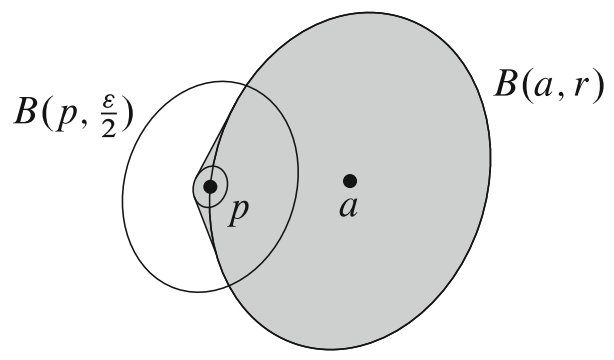

(also depending on $\varepsilon$ and on $\|\cdot\|)$ such that the following holds: If $a \in R_{i}$ with $r:=\operatorname{dist}\left(a, P_{i}\right) \leq s_{0}$ and $p \in P_{i}$ is a point attaining the distance $\operatorname{dist}\left(a, P_{i}\right)$, then the set

$$
K:=\operatorname{conv}(\{a\} \cup \mathrm{B}(p, \rho))
$$

is contained in $R_{i}$.

Proof As in the Euclidean case, we begin by observing that $a \in \operatorname{dom}\left(p, \bigcup_{j \neq i} S_{j}\right)$ and also $\mathrm{B}\left(p, \frac{\varepsilon}{4}\right) \subseteq \operatorname{dom}\left(p, \bigcup_{j \neq i} S_{j}\right)$ by Observation 2.2. Thus, the set $D:=\mathrm{B}(a, r) \cup$ $\mathrm{B}\left(p, \frac{\varepsilon}{2}\right)$ is contained in the closure of $\mathbb{R}^{d} \backslash \bigcup_{j \neq i} S_{j}$. We now want to find an open convex subset $C \subseteq D$ such that $a$ and $B(p, \rho)$ are contained in $\operatorname{dom}\left(p, \mathbb{R}^{d} \backslash C\right)$, since the latter region is convex by Lemma 4.1 and thus it contains $K$ as well.

We let $C$ be the interior of $\operatorname{conv}(B(a, r) \cup B(p, 2 \rho))$ with $\rho$ sufficiently small (the restrictions on it will be apparent from the proof below); see Fig. 11. It is clear that $\{a\} \cup B(p, \rho) \subseteq \operatorname{dom}\left(p, \mathbb{R}^{d} \backslash C\right)$, and so it remains to prove $C \subseteq D$.

To this end, it is sufficient to prove the following: If $B:=\mathrm{B}(0,1)$ is the unit ball of $\|\cdot\|$ and $\eta>0$ is given, then there exists $\delta>0$ such that for every $x \in \mathbb{R}^{d}$ with $\|x\| \leq 1+\delta$, the "cap" $\operatorname{conv}(B \cup\{x\}) \backslash B$ has diameter at most $\eta$. This is a wellknown and easily proved property of uniformly convex norms. (Proof sketch: If $x$ with $\|x\|=1+\delta$ has a cap of large diameter, then there is $z$ of norm 1 and half of the diameter away from $x$ such that the line $x z$ avoids the interior of $B$. Let $y$ be the other intersection of this line with $\partial \mathrm{B}(0,1+\delta)$ - then $x y$ is a long segment that cuts in $\mathrm{B}(0,1+\delta)$ into depth only $\delta$.)

Proof (Proof of Theorem 1.2) The overall strategy of the proof is exactly as for Theorem 1.1 (see Sect. 3). The constant $\alpha$ in (A) may also depend on the considered norm $\|\cdot\|$. This quantification also needs to be added in the appropriate version of Lemma 3.2.

In the proof of that lemma, the first place where we use a property not shared by all norms is below (2); we need that the triangle inequality may hold with equality only for collinear points - this remains true for all rotund norms.

Then we proceed as in the Euclidean case, introducing the the cone $K=\operatorname{conv}(\{a\} \cup$ $B(p, \rho))$ as in Lemma 4.2. There is some $\gamma>0$, depending on $\varepsilon, s_{0}$, and the norm $\|\cdot\|$, such that the appropriate Euclidean cone with opening angle $\gamma$ is contained in $K$. (Here and in the sequel we implicitly use the fact that every norm on $\mathbb{R}^{d}$ is between 
two constant multiples of the Euclidean norm, which is well known and immediate by compactness. All angles are measured in the usual Euclidean sense.)

We define the rays $k$ and $l$, again following the Euclidean proof. For the next step, we need that, since the angle between these rays is bounded away from the straight angle, at least one of the rays $k, l$ cuts a segment of significant length $\beta$ from the ball $\mathrm{B}\left(b^{\prime}, r\right)$. Instead of an explicit formula as in the Euclidean case, we use the uniform smoothness of the norm (formula (1) in Sect. 1); thus, $\beta$ also depends on the modulus of smoothness of the norm. The rest of the proof goes through unchanged.

\section{Non-uniqueness examples}

As we saw in the introduction, two point sites with the same $x$-coordinate have at least two zone diagrams under the $\ell_{1}$ metric. Here we show that only the non-smoothness (sharp corners) of the $\ell_{1}$ unit ball is essential for this example, while the straight edges can be replaced by curved ones.

Proposition 5.1 There exists a rotund norm in the plane, arbitrarily close to the $\ell_{1}$ norm, such that two distinct point sites with the same $x$-coordinate have (at least) two different zone diagrams.

The appropriate norm is not difficult to describe, but proving the non-uniqueness of the zone diagram is more demanding, since it seems hard to find an explicit description of a zone diagram for non-polygonal norms.

Informally, we construct the desired norm by slightly "inflating" the unit ball of the planar $\ell_{1}$ norm, so that the edges bulge out and the norm becomes rotund. It is important that the inflation is asymmetric, as is schematically indicated in Fig. 12 (in the "real" example we inflate much less). We will denote the resulting norm by $\|\cdot\|_{(1)}$; the subscript should remind the reader of "inflated $\ell_{1}$ " graphically.

To explain the purpose of the asymmetry in our example, we consider the bisector of the points $p=(-1,1)$ and $q=(1,-1)$, i.e., the set of all points equidistant to $p$ and $q$. For the $\ell_{1}$ norm, the bisector is "fat", as shown in Fig. 13 left-it consists of a segment and two quadrants. By a small inflation, which makes the norm rotund, the

Fig. 12 A schematic illustration of the unit ball of $\|\cdot\|_{(1)}$

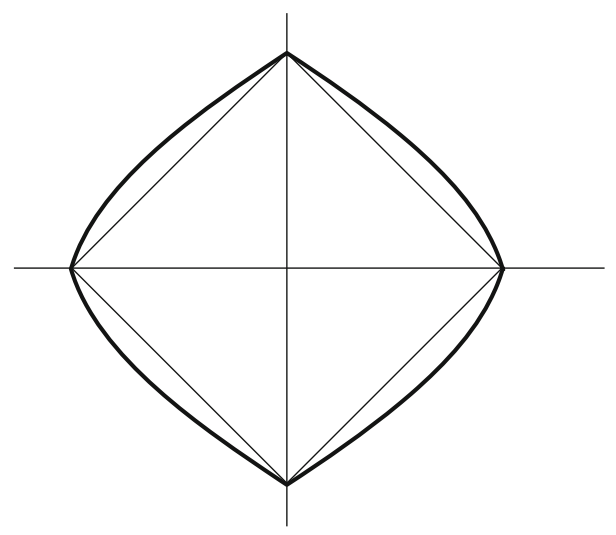



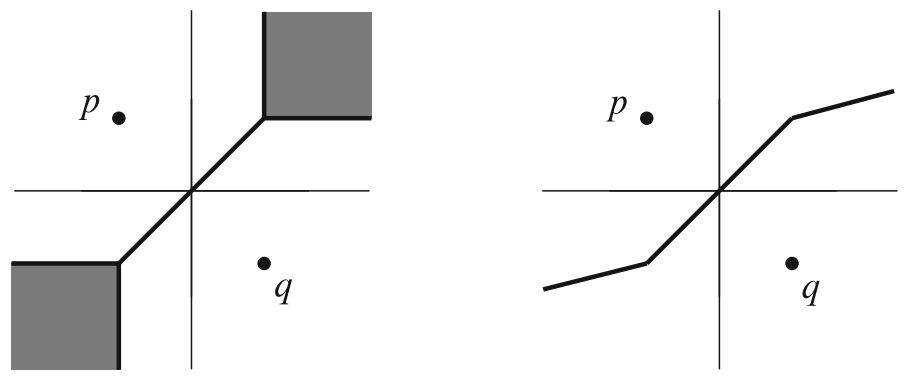

Fig. 13 The bisector of $p$ and $q$ under the $\ell_{1}$ norm and under $\|\cdot\|_{(1)}$ (schematic)
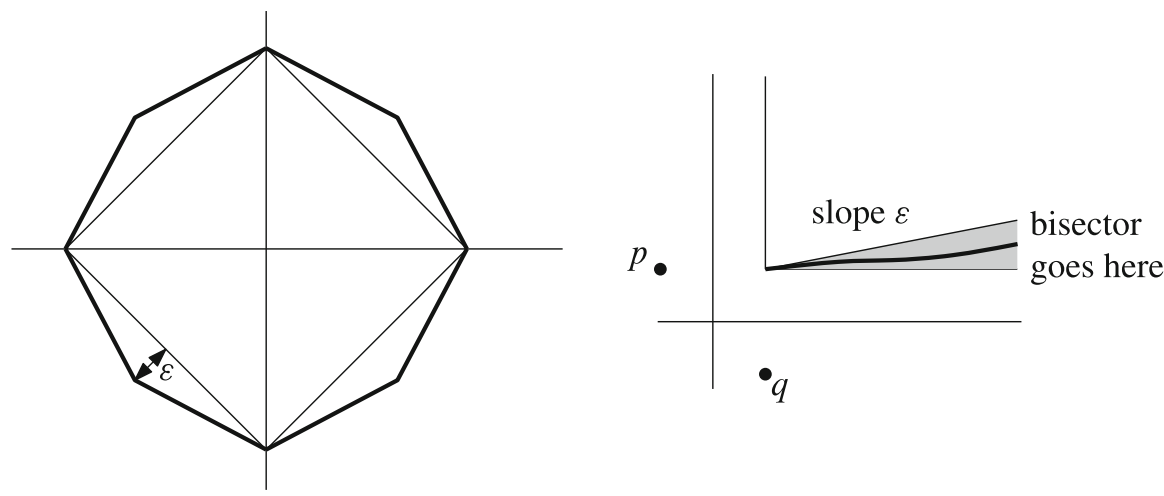

Fig. 14 The conditions in Lemma 5.2

middle segment of the bisector is changed only very slightly, but the "ambiguity" of the $\ell_{1}$ bisector in the quadrants is "resolved", and the quadrants collapse to (possibly curved) rays. Now if the inflation were symmetric, we would get straight rays with slope 1 in the bisector, but with an asymmetric inflation, we can get a (positive) slope as small as we wish.

In order to establish the required properties of the bisector formally, a safe route (if perhaps not the most conceptual one) is to describe $\|\cdot\|_{(1)}$ analytically. The rays of the bisector will be slightly curved rather than straight, but for the zone diagram construction this will do as well.

Lemma 5.2 For every $\varepsilon>0$ there exists a rotund norm $\|\cdot\|_{(1)}$ in the plane, whose unit ball contains the $\ell_{1}$ unit ball and is contained in the octagon as in Fig. 14 left, such that the portion of the bisector of the points $p=(-1,1)$ and $q=(1,-1)$ lying in the quadrant $\{(x, y): x, y \geq 1\}$ is an $x$-monotone curve lying below the line $y=\varepsilon(x-1)+1$ (Fig. 14 right).

Proof The construction has two positive parameters, $\alpha$ and $\delta$, where $\alpha$ is small and $\delta$ is still much smaller.

We let $\|\cdot\|^{\prime}$ be the Euclidean norm scaled by $\alpha$ in the horizontal direction; that is, $\|(x, y)\|^{\prime}=\sqrt{\alpha^{2} x^{2}+y^{2}}$. Let $\|\cdot\|^{\prime \prime}$ be the $\ell_{1}$ norm scaled by a suitable factor $\beta$ (close 
to 1$)$ in the vertical direction: $\|(x, y)\|^{\prime \prime}=|x|+\beta|y|$. The norm $\|\cdot\|_{(1)}$ is obtained as $a^{\prime}\|\cdot\|^{\prime}+a^{\prime \prime}\|\cdot\|^{\prime \prime}$, where $a^{\prime}, a^{\prime \prime}>0$ are suitable coefficients. This obviously yields a norm, which is rotund since $\|\cdot\|^{\prime}$ is rotund.

We want the contribution of $\|\cdot\|^{\prime}$ to be small compared to that of $\|\cdot\|^{\prime \prime}$, and that the corners of the unit ball of $\|\cdot\|_{(1)}$ coincide with those of the $\ell_{1}$ unit ball. This finally leads to the formula

$$
\|(x, y)\|_{(1)}:=\delta \sqrt{\alpha^{2} x^{2}+y^{2}}+(1-\alpha \delta)|x|+(1-\delta)|y| .
$$

Figure 12 is actually obtained from this formula with $\delta=0.7$ and $\alpha=0.5$. It is easy to check that, as the picture suggests, $\|\cdot\|_{(1)} \leq\|\cdot\|_{1}$ (and thus the $\ell_{1}$ unit ball is contained in the $\|\cdot\|_{(1)}$ unit ball), and for $\delta$ is sufficiently small in terms of $\alpha$ and $\varepsilon$, the unit ball of $\|\cdot\|_{(1)}$ is contained in the octagon as in the lemma.

It remains to investigate the bisector of $p$ and $q$ for $x \geq 1$ and $y \geq 1$. For convenience, we translate $p$ and $q$ by $(-1,-1)$ and scale by $\frac{1}{2}$. Then the bisector is given by the equation $\|(x+1, y)\|_{(1)}=\|(x, y+1)\|_{(1)}$, with the region of interest being the positive quadrant $x, y \geq 0$. For $x, y \geq 0$, the absolute values can be removed, $\delta$ disappears from the equation, and we obtain $\sqrt{\alpha^{2}(x+1)^{2}+y^{2}}+1-\alpha=$ $\sqrt{\alpha^{2} x^{2}+(y+1)^{2}}$. This can be solved for $y$ explicitly, with the only positive root

$$
y=\frac{1-\alpha}{2-\alpha}\left(\sqrt{1+2 \alpha x+2 \alpha x^{2}}-1+\frac{\alpha}{1-\alpha} x\right) .
$$

This is the equation of the bisector curve in the positive quadrant. It is a simple exercise in calculus (distinguishing the cases $\alpha x \leq 1$ and $\alpha x>1$, say) to show that $y \leq C \sqrt{\alpha} x$ for all $x>0$ and all sufficiently small $\alpha$ (here $C$ is a suitable constant).

Proof (Proof of Proposition 5.1) We show that the zone diagram of the sites $p^{-}=$ $(0,-1)$ and $p^{+}=(0,+1)$ under the norm $\|\cdot\|_{(1)}$ as in the lemma, with $\varepsilon$ sufficiently small, is not unique.

First we consider the zone diagram only inside the vertical strip $V:=[-2,2] \times \mathbb{R}$. Let $R_{0}^{+}$be the region as in Fig. 15, i.e., the part of the region of $p^{+}$within $V$ in an

Fig. 15 The regions $R_{0}^{+}, S_{0}^{+}, R_{0}^{-}, S_{0}^{-}$in the vertical strip $V$

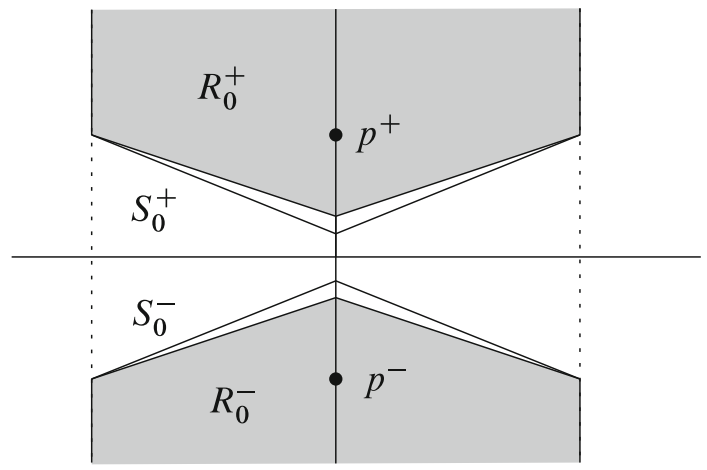


Fig. 16 The region $\tilde{S}^{+}$defined using bisectors, and a region containing $\tilde{R}^{-}$

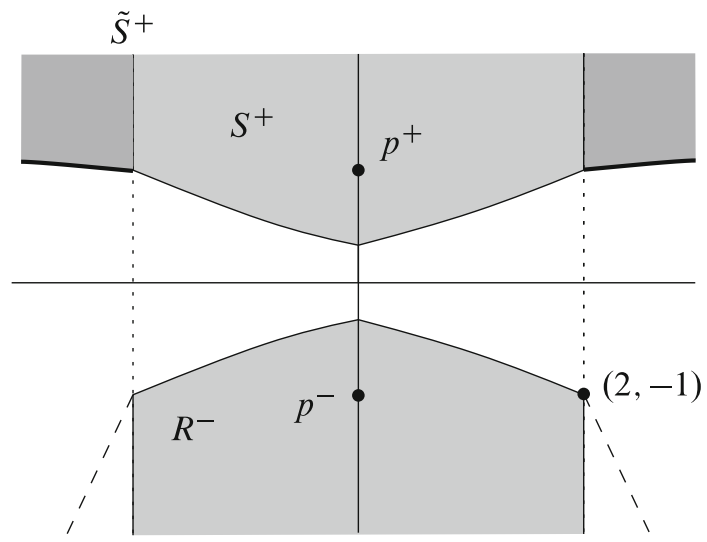

$\ell_{1}$ zone diagram of $p^{-}, p^{+}$. Let $S_{0}^{+}$be obtained by pulling the bottom vertex of $R_{0}^{+}$ downward by $\eta$ (which is another small positive parameter), and let $R_{0}^{-}, S_{0}^{-}$be the reflections of $R_{0}^{+}, S_{0}^{+}$by the $x$-axis.

Let us consider the region $\operatorname{dom}\left(p^{-}, R_{0}^{+}\right)$inside $V$ (distances measured by our norm $\left.\|\cdot\|_{(1)}\right)$. For every point $x \in V$ below $R_{0}^{+}$, the $\|\cdot\|_{(1)}$-distance to $R_{0}^{+}$coincides with the $\ell_{1}$ distance, which is simply the length of the vertical segment from $x$ to $\partial R_{0}^{+}$. From this it is clear that $\operatorname{dom}\left(p^{-}, R_{0}^{+}\right) \supseteq R_{0}^{-}$(since $R_{0}^{-}$is the dominance region of $p^{-}$against $R_{0}^{+}$in the $\ell_{1}$ metric, and $\left.\|\cdot\|_{(1)} \leq\|\cdot\|_{1}\right)$. Moreover, it's easy to check that for $\varepsilon$ (the parameter controlling the choice of $\|\cdot\|_{(1)}$ ) sufficiently small, we also have $\operatorname{dom}\left(p^{-}, R_{0}^{+}\right) \subseteq S_{0}^{-}$.

Thus, we have $R_{0}^{-} \subseteq \operatorname{dom}\left(p^{-}, R_{0}^{+}\right) \subseteq S_{0}^{-}$, and by the vertical symmetry we also get $R_{0}^{+} \subseteq \operatorname{dom}\left(p^{+}, R_{0}^{-}\right) \subseteq S_{0}^{+}$. Arguing as in the proof of Theorem 2.1, we get that there exist regions $R^{+}, R^{-}, S^{+}, S^{-}$, where $R^{-}$is the reflection of $R^{+}, S^{-}$is the reflection of $S^{+}$, such that $R_{0}^{-} \subseteq R^{+} \subseteq S^{+}$, and $\left(R^{-}, S^{+}\right)$is a zone diagram of $\left(p^{-}, p^{+}\right)$(and so is $\left(S^{-}, R^{+}\right)$, but we actually have $R^{+}=S^{+}$, although we will neither need this nor prove it).

All of this refers to the vertical strip $V$ (so, formally, the metric space in these arguments is $V$ with the $\|\cdot\|_{(1)}$ metric). Now we move on to the full plane $\mathbb{R}^{2}$, and we let $\tilde{S}^{+}$be the region consisting of $S^{+}$plus two parts of the upper halfplane outside $V$ as in Fig. 16: The right part is delimited by a part of the bisector of $p^{+}$and $(2,-1)$ (drawn thick), and the left part by a part of the bisector of $p^{+}$and $(-2,-1)$.

Now we set $\tilde{R}^{-}:=\operatorname{dom}\left(p^{-}, \tilde{S}^{+}\right)$. The distance of points inside $V \backslash S^{+}$to $\tilde{S}^{+}$is still the vertical distance, i.e., the same as the distance to $S^{+}$, and so $\tilde{R}^{-} \cap V=R^{-}$. For the part of $\tilde{R}^{-}$outside $V$, we don't need an exact description-it is sufficient that it lies below the dashed rays in Fig. 16 (using the property of the bisectors as in Lemma 5.2, one can see that these rays can be taken as steep as desired, by setting $\varepsilon$ sufficiently small). From this we can see that for every point of the upper halfplane on the right of $V$, the nearest point of $\tilde{R}^{-}$is the corner $(2,-1)$.

Therefore, $\operatorname{dom}\left(p^{+}, \tilde{R}^{-}\right)=\tilde{S}^{+}$, and hence $\left(\tilde{R}^{-}, \tilde{S}^{+}\right)$is a zone diagram of $\left(p^{-}, p^{+}\right)$. But the mirror reflection of this zone diagram about the $x$-axis yields another, different zone diagram. 


\section{Smooth norms in the plane}

Proposition 5.1 showed that the assumption of smoothness in Theorem 1.2 cannot be dropped, even for the simplest case of two singleton sites in the plane. Theorem 1.3, which we will prove here, states that the rotundity assumption can be dropped in this special case.

Smoothness of the norm means that a metric ball has a unique supporting halfspace at every point of its boundary. Thus, for a nonzero vector $a$, we can define $\top_{a}^{>}$to be the open halfspace that touches (but does not intersect) the ball $\mathrm{B}(-a,\|a\|)$ at the origin. We write $\top_{a}^{\bar{a}}$ for the boundary of $\top_{a}^{>}$and let $\top_{\vec{a}}^{\vec{a}}=\top_{a}^{>} \cup \top_{\bar{a}}$. For nonzero vectors $a$ and $b$, define $a \sim b$ when $\top_{a}^{>}=\top_{b}^{>}$. Then $\sim$ is an equivalence relation. It is easy to see (Fig. 17) that for nonzero vectors $a_{1}, \ldots, a_{m}$, we have

$$
\left\|a_{1}+\cdots+a_{m}\right\|=\left\|a_{1}\right\|+\cdots+\left\|a_{m}\right\| \quad \text { if and only if } \quad a_{1} \sim \cdots \sim a_{m} .
$$

Lemma 6.1 Let $\|\cdot\|$ be a smooth norm on $\mathbb{R}^{d}$. Then there is a positive number $\beta$ such that for any unit vectors $u, v$ with $\|u+v\|>2-\beta$, we have $\|u-\beta v\| \leq 1$.

Proof For unit vectors $u$ and $v$ with $v \in \top_{u}^{>}$, let $\alpha_{u, v}$ be the length of the part of the line $u+\mathbb{R} v$ that lies inside the unit ball. Thus $\alpha_{u, v}$ is the unique positive number with $\left\|u-\alpha_{u, v} v\right\|=1$. Since $\alpha_{u, v}$ is continuous in $u$ and $v$, so is $f(u, v)=$ $\max \left\{\alpha_{u, v}, 2-\|u+v\|\right\}$. We extend $f$ by setting $f(u, v)=2-\|u+v\|>0$ for $v \notin \top_{u}^{>}$. Since $f$ is a lower semi-continuous function defined for all pairs of unit vectors, it attains a minimum. Let $\beta$ be this minimum.

Lemma 6.2 Let $\|\cdot\|$ be a smooth norm on $\mathbb{R}^{d}$. For any $\kappa>0$, there is $\varepsilon>0$ such that, for any vectors $u, v$ with $\|u\|,\|v\| \geq 1$ and $\|u-v\|<\varepsilon$, we have $\operatorname{dist}(y, \mathrm{~B}(u,\|u\|)) \leq$ $\kappa\|y\|$ for any $y \in \mathrm{B}(v,\|v\|)$.

Proof Since $\operatorname{dist}(y, \mathrm{~B}(u,\|u\|)) \leq 2 \varepsilon$, it is clear that, for any constant $\eta>0$, the claim holds if we consider only those $y$ with $\|y\| \geq \eta$. Therefore, it suffices to prove the existence of $\eta>0$, depending on $\|\cdot\|$ and $\kappa$, such that the claim holds for any $y$ with $\|y\|<\eta$.

We find the desired $\eta$ and $\varepsilon$ as follows (Fig. 18). Since the norm is smooth, the surface of a ball looks like a hyperplane locally at each point. Thus, there exists $\eta>0$
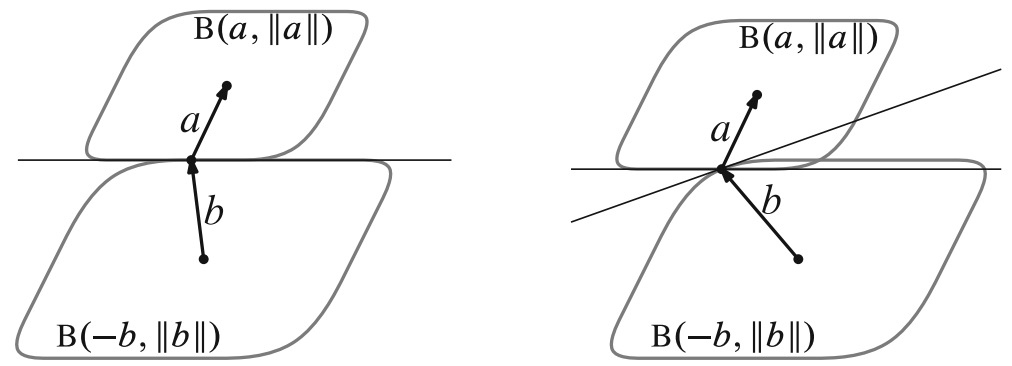

Fig. $17\|a+b\|=\|a\|+\|b\|$ if and only if $a \sim b$ (Eq. (3) with $m=2$ ) 


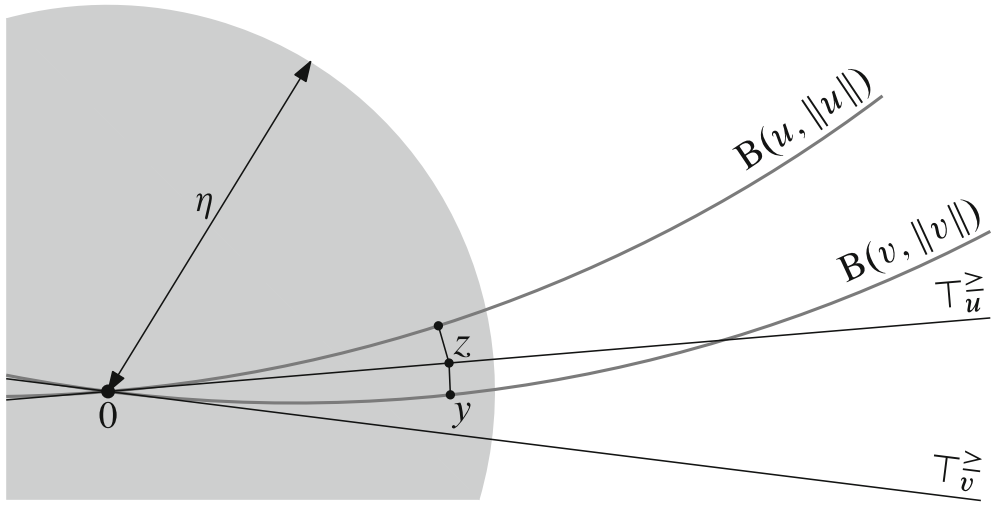

Fig. 18 When $u$ and $v$ are close, $y \in \mathrm{B}(v,\|v\|)$ is not very far from $\mathrm{B}(u,\|u\|)$

Fig. 19 The conclusion of Lemma 6.3 states that $\operatorname{dom}(v, u)$ and the boundary of $\mathrm{B}(u, 1)$ "make a positive angle" at the origin. We prove this by showing that there is a cone (shaded) whose axis is the tangent vector $w$ and which does not overlap $\operatorname{dom}(v, u)$

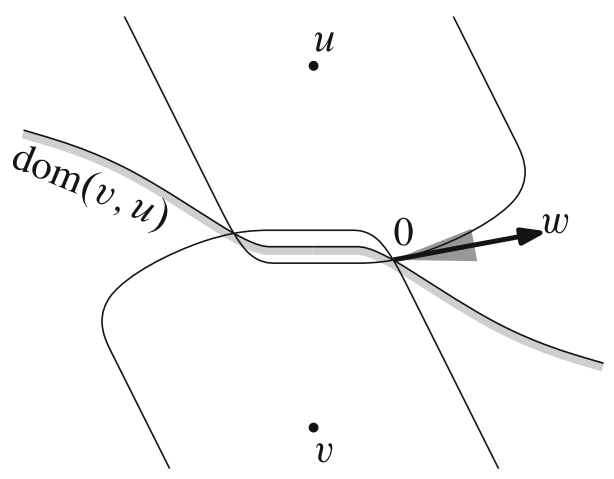

such that for any $u \in \mathbb{R}^{d}$ with $\|u\| \geq 1$ and any $z \in \top \geq{ }_{u}$ with $\|z\|<\eta(1+\kappa / 2)$, we have $\operatorname{dist}(z, \mathrm{~B}(u,\|u\|)) \leq \kappa\|z\| /(2+\kappa)$. Also, since changing slightly a vector $u$ with $\|u\| \geq 1$ moves $\top_{\vec{u}}^{\geq}$only slightly, there is $\varepsilon>0$ so small that for any vectors $u, v$ with $\|u\|,\|v\| \geq 1$ and $\|u-v\|<\varepsilon$, we have $\operatorname{dist}\left(y, \top_{u}^{\geq}\right) \leq \kappa\|y\| / 2$ for all $y \in \top_{v}^{\geq}$.

Since $y \in \mathrm{B}(v,\|v\|) \subseteq \top \geq$, we have $\operatorname{dist}\left(y, \mathrm{~T}_{\bar{u}}\right) \leq \kappa\|y\| / 2$ by our choice of $\varepsilon$. Let $z \in \top_{\bar{u}}^{\geq}$be a point attaining this distance. Since $\|z\| \leq\|y\|+\|z-y\| \leq\|y\|+$ $\kappa\|y\| / 2=\|y\|(1+\kappa / 2) \leq \eta(1+\kappa / 2)$, we have $\operatorname{dist}(z, \mathrm{~B}(u,\|u\|)) \leq \kappa\|z\| /(2+\kappa) \leq$ $\kappa\|y\| / 2$ by our choice of $\eta$. These imply $\operatorname{dist}(y, \mathrm{~B}(u,\|u\|)) \leq \kappa\|y\|$ by the triangle inequality.

Lemma 6.3 Let $\|\cdot\|$ be a smooth norm on $\mathbb{R}^{2}$. For unit vectors $u$ and $v$ with $\|u-v\|<2$, there is $\kappa>0$ such that for all $y \in \operatorname{dom}(v, u) \backslash \mathrm{B}(v, 1)$ sufficiently close to the origin (Fig. 19), $\operatorname{dist}(y, \mathrm{~B}(u, 1))>\kappa\|y\|$.

Proof Because $\|u-v\|<2$, the vectors $u$ and $-v$ do not share the supporting halfspace. Therefore, there is a (unique) unit vector $w \in \top_{u}=$ that heads out of $\mathrm{B}(v, 1)$. Since

$$
\lim _{\delta \rightarrow 0} \frac{\|u-\delta w\|-1}{\delta}=0, \quad \beta:=\lim _{\delta \rightarrow 0} \frac{\|v-\delta w\|-1}{\delta}>0,
$$


there exists $\delta_{0}>0$ so small that for all positive $\delta<\delta_{0}$, we have

$$
\frac{\|u-\delta w\|-1}{\delta}<\frac{1}{3} \beta, \quad \frac{\|v-\delta w\|-1}{\delta}>\frac{2}{3} \beta,
$$

and hence $\|u-\delta w\|<\|v-\delta w\|-\beta \delta / 3$. This implies that $\|u-x\|<\|v-x\|$ for all $x \in \mathrm{B}(\delta w, \beta \delta / 6)$. Thus, $\operatorname{dom}(v, u)$ is disjoint from a cone (except at the origin) whose vertex is at the origin and axis is the vector $w$ (see Fig. 19). This implies the assertion of the lemma.

Now we look at the situation of Theorem 1.3. Let $\mathbf{R}=\left(R_{0}, R_{1}\right)$ and $\mathbf{S}=\left(S_{0}, S_{1}\right)$ be pairs as in Theorem 2.1. As before, it suffices to show that $\mathbf{R}=\mathbf{S}$. Suppose otherwise. Then $h=\min \left\{\operatorname{dist}\left(p_{0}, S_{0} \backslash R_{0}\right), \operatorname{dist}\left(p_{1}, S_{1} \backslash R_{1}\right)\right\}$ exists.

Lemma 6.4 In the above setting, if a point $c \in \overline{S_{0} \backslash R_{0}}$ satisfies $\left\|c-p_{0}\right\|=h$, then

(a) $\left\|c-p_{1}\right\|=2 h$;

(b) there is a point $c^{\prime} \in \overline{S_{1} \backslash R_{1}}$ satisfying $\left\|c^{\prime}-c\right\|=\left\|c^{\prime}-p_{1}\right\|=h$.

Proof Note that $c \in R_{0}$, since otherwise $S_{0} \backslash R_{0}$ intersects a part of the segment $c p_{0}$ of positive length, contradicting the minimality of $h$.

There is a sequence $\left(x_{i}\right)_{i \in \mathbb{N}}$ of points in $S_{0} \backslash R_{0}$ that converges to $c$. For each $i \in \mathbb{N}$, let $y_{i} \in S_{1}$ be a closest point to $x_{i}$. Since $x_{i} \in S_{0} \backslash R_{0}$, we have $\left\|y_{i}-x_{i}\right\|=$ $\operatorname{dist}\left(x_{i}, S_{1}\right)<\left\|p_{0}-x_{i}\right\|$ and $y_{i} \in S_{1} \backslash R_{1}$. The sequence $\left(y_{i}\right)_{i \in \mathbb{N}}$ has a subsequence $\left(y_{j_{i}}\right)_{i \in \mathbb{N}}$ that converges to a point $c^{\prime} \in \overline{S_{1} \backslash R_{1}}$ (Fig. 20). Note that

$$
\left\|c^{\prime}-p_{1}\right\| \leq\left\|c-c^{\prime}\right\|=\lim _{i \rightarrow \infty}\left\|x_{j_{i}}-y_{j_{i}}\right\| \leq \lim _{i \rightarrow \infty}\left\|p_{0}-x_{j_{i}}\right\|=\left\|p_{0}-c\right\|=h,
$$

where the first inequality is by $c^{\prime} \in S_{1}$ and $c \in R_{0}$. In fact, this holds in equality by the minimality of $h$. We have proved (b).

Fig. 20 Lemma 6.4

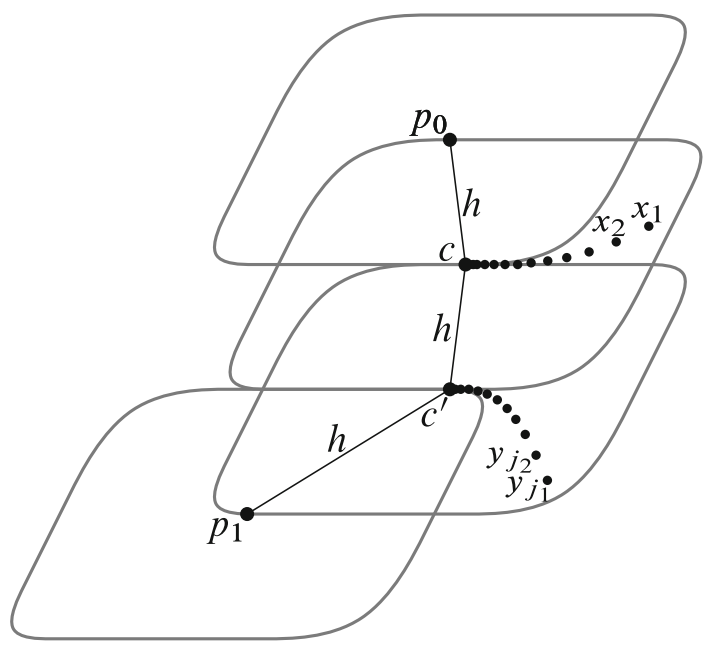


For each $i$, since $S_{1} \backslash R_{1}$ intersects a part of the segment $y_{j_{i}} c^{\prime}$ of positive length, $y_{j_{i}} \notin \mathrm{B}\left(p_{1}, h\right)$ by the minimality of $h$. Also, $y_{j_{i}} \in S_{1} \subseteq \operatorname{dom}\left(p_{1}, c\right)$. As $i$ increases, $y_{j_{i}}$ comes arbitrarily close to $c^{\prime}$. Hence, if (a) were not true, Lemma 6.3 would give a constant $\kappa>0$ such that $\operatorname{dist}\left(y_{j_{i}}, \mathrm{~B}(c, h)\right)>\kappa\left\|y_{j_{i}}-c^{\prime}\right\|$ for all but finitely many $i$. On the other hand, since $y_{j_{i}}$ is in $\mathrm{B}\left(x_{j_{i}},\left\|x_{j_{i}}-c^{\prime}\right\|\right)$ and $\left(x_{j_{i}}\right)_{i \in \mathbb{N}}$ converges to $c$, Lemma 6.2 shows that $\operatorname{dist}\left(y_{j_{i}}, \mathrm{~B}(c, h)\right) \leq \kappa\left\|y_{j_{i}}-c^{\prime}\right\|$ for all but finitely many $i$. This is a contradiction. We have proved (a).

Lemma 6.5 In the above setting, $\left\|p_{0}-p_{1}\right\|=3 h$.

Proof By the definition of $h$, there is a point $c \in \overline{S_{0} \backslash R_{0}}$ satisfying $\left\|c-p_{0}\right\|=h$. By Lemma 6.4(b), there is a point $c^{\prime} \in \overline{S_{1} \backslash R_{1}}$ satisfying $\left\|c^{\prime}-c\right\|=\left\|c^{\prime}-p_{1}\right\|=h$. By Lemma 6.4(a) (and the same lemma with the sites swapped), $\left\|c-p_{1}\right\|=\left\|c^{\prime}-p_{0}\right\|=$ $2 h$. This implies $\left(c-p_{0}\right) \sim\left(c^{\prime}-c\right) \sim\left(p_{1}-c^{\prime}\right)$ and thus $\left\|p_{0}-p_{1}\right\|=3 h$ by (3) at the beginning of this section.

To prove Theorem 1.3, we will construct a sequence $\left(b_{t}\right)_{t \in \mathbb{N}}$ of points in $R \backslash S$, as we did in Sect. 3. Recall that for each $i \in\{0,1\}$ and $b \in S_{i}$, we define $a(b)$ to be the closest point to $b$ that is in the intersection of $R_{i}$ with the segment $b p_{i}$ (note that since we do not have the cone lemma this time, the intersection of $b p_{i}$ and $\partial R_{i}$ is not always unique). As before, let $s(b)=\left\|b-p_{i}\right\|$ and $\delta(b)=\|b-a(b)\|$.

The proof goes as follows. This time, we begin with a point $b_{0} \in S_{0} \backslash R_{0}$ that is within distance $h+\varepsilon$ from the nearest site, for some small $\varepsilon>0$ (such a point $b_{0}$ exists by the definition of $h$ ), and take $b_{1}, b_{2}, \ldots$ as we did in Sect. 3 using Lemma 3.2: For each $b_{t} \in S_{i} \backslash R_{i}$, we let $b_{t+1} \in S_{1-i} \backslash R_{1-i}$ be a point that is at the same distance from $a\left(b_{t}\right)$ as $p_{i}$ is. Then each $b_{t}$ will be also within distance $h+\varepsilon$ from the nearest site $p_{i}$. Because we have proved that the sites are $3 h$ apart, and the path $p_{i}-a\left(b_{t}\right)-b_{t+1}-p_{1-i}$ consists of three segments shorter than $h+\varepsilon$, this path must be "almost straight". This implies that we will always have the case (B) in Sect. 3 (Fig. 7 left):

Lemma 6.6 In the above setting, the following holds for some $\varepsilon>0$ : For each $i \in\{0,1\}$ and $b \in S_{i} \backslash R_{i}$ satisfying $s:=s(b)<h+\varepsilon$, there is $b^{\prime} \in S_{1-i} \backslash R_{1-i}$ such that $\delta:=\delta(b), s^{\prime}:=s\left(b^{\prime}\right), \delta^{\prime}:=\delta\left(b^{\prime}\right)$ satisfy (B) of Sect. 3 (i.e., $\delta^{\prime} \geq \delta$ and $\left.s^{\prime} \leq s-\delta\right)$.

Proof Let $\varepsilon:=h \beta / 3$, where $\beta$ is as in Lemma 6.1. Let $b$ be as assumed. By the definition of $a:=a(b)$, there is $b^{\prime} \in S_{1-i}$ with $\left\|b^{\prime}-a\right\|=\left\|a-p_{i}\right\|$. We show that this $b^{\prime}$ qualifies. Since $s^{\prime}=\left\|b^{\prime}-p_{1-i}\right\| \leq\left\|b^{\prime}-a\right\|=\left\|a-p_{1-i}\right\|=s-\delta$, it suffices to prove that $\delta^{\prime} \geq \delta$ (which would then imply $b^{\prime} \notin R_{1-i}$ ).

By Lemma 6.5, we have

$$
\begin{aligned}
\left\|b^{\prime}-p_{i}\right\| & \geq\left\|p_{1-i}-p_{i}\right\|-\left\|p_{1-i}-b^{\prime}\right\|=3 h-s^{\prime}>3 h-s \geq 3 h-(h+\varepsilon) \\
& =2(h+\varepsilon)-3 \varepsilon \geq 2(h+\varepsilon)-\beta h>(h+\varepsilon)(2-\beta)>\left\|a-p_{i}\right\|(2-\beta) .
\end{aligned}
$$

By this and $\left\|b^{\prime}-a\right\|=\left\|a-p_{i}\right\|$, Lemma 6.1 yields $\left\|\left(b^{\prime}-a\right)-\beta\left(a-p_{i}\right)\right\| \leq\left\|a-p_{i}\right\|$. This remains true if we decrease $\beta$, since $\mathrm{B}\left(0,\left\|a-p_{i}\right\|\right)$ is convex. So we replace $\beta$ by $\|b-a\| /\left\|a-p_{i}\right\| \leq \varepsilon / h \leq \beta / 3<\beta$, obtaining $\left\|b^{\prime}-b\right\|=\left\|\left(b^{\prime}-a\right)-(b-a)\right\| \leq$ $\left\|a-p_{i}\right\|$. 
Since $b$ is in $S_{i}$ and $a^{\prime}:=a\left(b^{\prime}\right)$ is in $R_{1-i}$, we have $\left\|a^{\prime}-b\right\| \geq s$. Hence, $\delta^{\prime}=$ $\left\|b^{\prime}-a^{\prime}\right\| \geq\left\|a^{\prime}-b\right\|-\left\|b^{\prime}-b\right\| \geq s-\left\|a-p_{i}\right\|=\delta$, as desired.

The rest of the argument is similar to what we have already seen in Sect. 3 (and even simpler because we do not have case (A) this time): Starting at $b_{0} \in S \backslash R$ such that $s\left(b_{0}\right)<h+\varepsilon$, where $\varepsilon$ is as in Lemma 6.6, we define $b_{t+1}$, for each $t \in \mathbb{N}$, to be the point $b^{\prime}$ corresponding to $b=b_{t}$. By the lemma, $s\left(b_{t}\right)$ always decreases by at least $\delta\left(b_{0}\right)$, leading to a contradiction. This proves Theorem 1.3.

Acknowledgments We are grateful to Tetsuo Asano for valuable discussions including those on nonuniqueness examples for convex polygonal distances. We also express our gratitude to Daniel Reem for careful reading of and useful suggestions on the manuscript. Finally, we remark that the warm comments from the audience of our preliminary announcement of partial results at the 25th European Workshop on Computational Geometry (EuroCG 2009) encouraged us to work further. Some of the results in this paper were also presented at the 26th Annual Symposium on Computational Geometry (SoCG 2010). Part of this work was done while A.K. was visiting ETH Zürich, whose support and hospitality are gratefully acknowledged. His work was also supported by the Nakajima Foundation and the Natural Sciences and Engineering Research Council of Canada when he was a graduate student at the University of Toronto. The part of this research by T. T. was partially supported by the JSPS Grant-in-Aid for Scientific Research (B) 18300001.

\section{References}

1. Asano, T., Kirkpatrick, D.: Distance trisector curves in regular convex distance metrics. In: Proceedings of the 3rd International Symposium on Voronoi Diagrams in Science and Engineering, pp. 8-17. IEEE Computer Society, California (2006)

2. Asano, T., Matoušek, J., Tokuyama, T.: Zone diagrams: existence, uniqueness, and algorithmic challenge. SIAM J. Comput. 37(4), 1182-1198 (2007)

3. Asano, T., Matoušek, J., Tokuyama, T.: The distance trisector curve. Adv. Math. 212(1), 338-360 (2007)

4. Aurenhammer, F.: Voronoi diagrams - a survey of a fundamental geometric data structure. ACM Comput. Surv. 23(3), 345-405 (1991)

5. Benyamini, Y., Lindenstrauss, J.: Nonlinear Functional Analysis, vol. I, Colloquium Publications 48. American Mathematical Society (AMS), Providence (2000)

6. de Biasi, S.C., Kalantari, B., Kalantari, I.: Maximal zone diagrams and their computation. In: Proceedings of the 7th International Symposium on Voronoi Diagrams in Science and Engineering, pp. 171-180. IEEE Computer Society, California (2010)

7. Chun, J., Okada, Y., Tokuyama, T.: Distance trisector of a segment and a point. Interdiscip. Inf. Sci. 16(1), 119-125 (2010)

8. Kadets, M.I., Levitan, B.M.: Banach space. In: Hazewinkel, M. (Ed.) Encyclopedia of Mathematics. Springer, Berlin (2002). http://eom.springer.de/b/b015190.htm

9. Kawamura, A., Matoušek, J., Tokuyama, T.: Zone diagrams in Euclidean spaces and in other normed spaces. arXiv:0912.3016v1 (Preprint, 2009)

10. Kopecká, E., Reem, D., Reich, S.: Zone diagrams in compact subsets of uniformly convex normed spaces. Israel J. Math. (2011). doi:10.1007/s11856-011-0094-5

11. Okabe, A., Boots, B., Sugihara, K., Chiu, S.N.: Spatial Tessellations: Concepts and Applications of Voronoi Diagrams. Probability and Statistics, 2nd edn. Wiley, New York (2000)

12. Reem, D., Reich, S.: Zone and double zone diagrams in abstract spaces. Colloquium Mathematicum 115(1), 129-145 (2009)

13. Tarski, A.: A lattice-theoretical fixpoint theorem and its applications. Pac. J. Math. 5, 285-309 (1955) 